\title{
Numerical analysis of vibration-isolating effect of vibration-isolating slot under buried pipe subjected to millisecond blasting
}

\author{
Chong $\mathrm{Ji}^{1}$, Fuyin $\mathrm{Gao}^{2}$, Xinghua $\mathrm{Li}^{3}$, Yang $\mathrm{Yu}^{4}$, Liangyu Cheng ${ }^{5}$ \\ College of Field Engineering, The Army Engineering University of PLA, Nanjing, 210007, China \\ ${ }^{2}$ Corresponding author \\ E-mail:12468645816@qq.com, ${ }^{2472564948 @ q q . c o m,{ }^{3} 379523589 @ q q . c o m,{ }^{4} 962823438 @ q q . c o m,}$ \\ 5clychmy@qq.com
}

Received 11 September 2018; accepted 14 November 2018

DOI https://doi.org/10.21595/vp.2018.20213

Check for updates

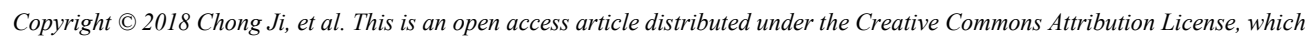
permits unrestricted use, distribution, and reproduction in any medium, provided the original work is properly cited.

\begin{abstract}
Research on vibration-isolating effects of vibration-isolating slot on buried pipe can be done by numerical method, without being disturbed by external environmental factors. It has measured data without relatively high experiment cost and analyzed the influence of some key parameters according to the results of numerical simulation. The results show that the vibration speed of the pipeline with vibration-isolating slot tends to have a larger decrease than those without vibration-isolating slot. What's more, the homogeneous explosive charge is discrepant in different working conditions, but the vibration-isolating ratio is similar in the vibration-isolating slot with same structure parameter. The millisecond blasting is hardly affected by total explosive charge. But the blasting seismic intensity is influenced by explosive charge in each stage directly.
\end{abstract}

Keywords: millisecond blasting, vibration-isolating effect, vibration-isolating slot, numerical simulation, vibration.

\section{Introduction}

The explosive charge structure can be classified into concentrated charge and dispersive charge. Although the experts agreed that the two charge structures have different effects on the vibration effect of blasting earthquake through the long-term experiment, its internal mechanism, especially the transmission of vibration and energy in dispersive charge, hasn't come to a clear conclusion. But the exploration of differential blasting keeps going in the Academia all the time. $\mathrm{Wu}$ Congshi et al. [1] analyzed the connection between millisecond blasting and the blasting vibration, concluding the appropriate interval of millisecond blasting in their own research project. Lin Dachao and Zhang Qi et al. [2] designed experiment to research millisecond blasting and blasting vibration. Namely, under $20 \mathrm{~ms}, 40 \mathrm{~ms}, 60 \mathrm{~ms}$ and $80 \mathrm{~ms}$ delay time and distance from the explosion point $10 \mathrm{~m}, 20 \mathrm{~m}$ and $40 \mathrm{~m}$, the theoretical analysis and research on the vibration velocity and the rule of the power spectrum arrangement were studied by testing the vibration velocity on the direction vertical to the ground. According to the study, reducing ground vibration strength through millisecond blasting, there is an optimal value of delay time. Anderson [3] summarized the relationship between the delay time and the vibration mainly based on an immense amount of engineering blasting data, and expressed their relationships by gray scale. Gao Wenxue and Jin Qiankun [4] studied slot millisecond blasting of deep hole by the engineering practice and based on the study of the dynamic finite element program, and discussed the slot rock movement law and the inherent mechanism of millisecond blasting. It showed that, based on engineering practice, the dynamic situation of rock blasting fragmentation is really presented, which has become the support of blasting theory. Hewetra [5] studied underground structure stability under blasting vibration wave, analyzed influence of surrounding rocks with different properties on vibration velocity, and put forward effective vibration damping measures. Anony [6] used the finite element program to analyze the damage model of modified fragmentation effect on surrounding rock under the condition of different detonating modes, which is according to the 
dynamics theory. Li Xi-bing and Ling Tong-hua [7] utilized the piecewise linear interpolation method instead of analytical expression of trigonometric interpolation, which means less complication and higher accuracy in the calculation of single-segment and multi-segment millisecond blasting signal response spectrum. The results showed that single-segment and multi-segment has quite discrepant structure response to blasting vibration. In addition, the blasting seismic wave differences exist in the choice of amplification effects for the distinction of the structure, for instance, such millisecond blasting in the influence of the damping effect at the same time, increase the probability of structural response to selective amplification some of the vibrating body.

It is not a good way to do researches on vibration-isolating effect of vibration-isolating slot subjected to millisecond blasting by experiment, because blasting test cost is relatively high, the test site is special and it has a certain risk. At the same time, in view of the limited monitoring range, vibration-isolating effect of vibration-isolating slot in the actual test is also just passable. Through small blasting test in advance, it can inaccurately predict the propagation and attenuation of blasting seismic wave in the soil. As a result, direct field experiment may fail to acquire ideal effect, and even cause the waste of human resources, material and financial resources generated by more working conditions. In contrast, it is better to do research on vibration-isolating effect of vibration isolating by the numerical method without external environmental factors. It can measure data without the high experiment cost and analyze the influence of some key parameters according to the results. In the same condition, the numerical simulation cost much less than the test. Therefore, numerical simulation is an indispensable method to analyze the effect of the micro-differential blasting on shock absorbing.

\section{Calculation model}

The initial conditions of the numerical simulation model are determined according to the principle of maximum blasting damage effect. Considering the damage of buried pipe subjected to millisecond blasting for a certain distance from the blasting area, the calculation model is mainly produced by the homogeneous explosive charge. The distance between the charge and the axis of the pipeline is $50 \mathrm{~m}$, and the pipe buried $1.2 \mathrm{~m}$ underground.

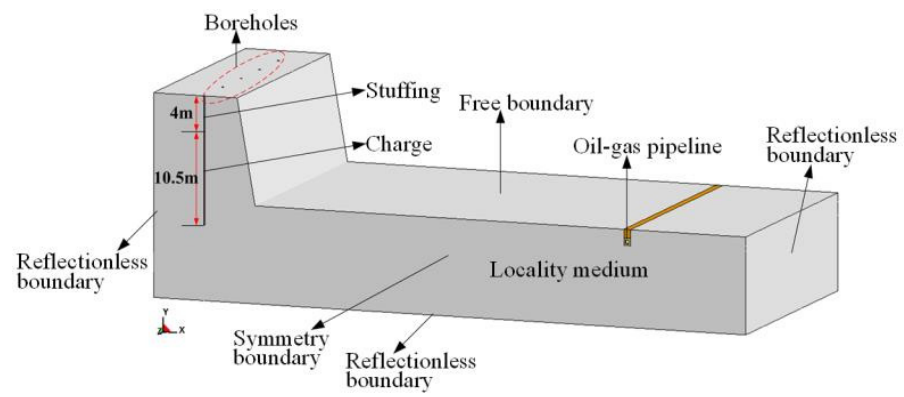

Fig. 1. The numerical calculation model

According to the characteristics of the problem studied, the coupling algorithm of multi material Euler and Lagrange structure can be applied to calculate the interaction between explosives, air and other solid structures. In the actual modeling process, it defines explosives and air as Euler grids, and ensures the common nodes among them. It defines the site medium, the fine sand in the pipe trench, the backfill soil medium and the steel pipe as the Lagrange mesh. The interaction between the fine sand in the trench and pipe adopts the surface-surface contact analysis. On contact surface in fine sand and steel pipe, steel pipe will take the master contact surface (Master Surface, for larger stiffness), and soil can be considered as the slave contact surface (Slave Surface, relatively soft). In order to improve the mesh accuracy and save computing resources, we only need to establish the $1 / 2$ model relative to the hole axis and adopt the $\mathrm{g}$ - $\mathrm{cm}$-s system. The 
meshes of explosives and surrounding rock are denser than others. Because the rock is affected by blasting seismic waves, which is in the range of elastic deformation, so the mesh could be sparse. The SOLID164 element is used to mesh the above three-dimensional numerical models. The numerical calculation model is shown in Fig. 1.

\section{Numerical simulation results and analysis}

Fig. 2, Fig. 3, Fig. 4 are the propagation of blasting seismic waves under the condition of no shock absorption. As the vibration velocity cloud of particle is applied, this part is more intuitionistic and clear. Fig. 2, Fig. 3 and Fig. 4 respectively represents $275 \mathrm{~kg}$ homogeneous explosive charge ( 1 hole), $1375 \mathrm{~kg}$ homogeneous explosive charge (5 holes) and $2475 \mathrm{~kg}$ homogeneous explosive charge ( 9 holes). The maximum vertical vibration data at the pipe location can be extracted through post-processing software. Fig. 5 is the maximum vertical vibration velocity history curve of the pipe under the condition of no vibration-isolating slot.

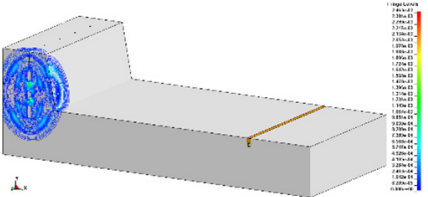

a) T1, 1/2 model

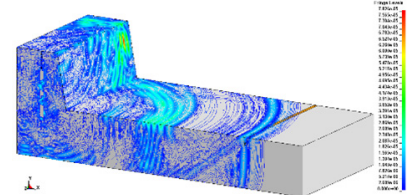

b) $\mathrm{T} 2,1 / 2$ model

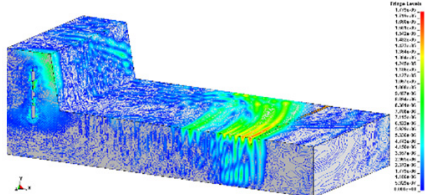

c) T3, 1/2 model

Fig. 2. The propagation of blasting seismic waves ( $275 \mathrm{~kg}$ charge per delayed interval)

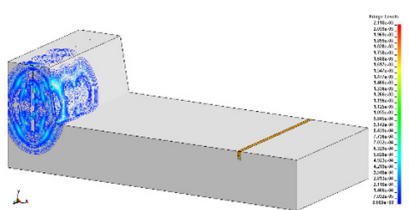

a) T1, 1/2 model

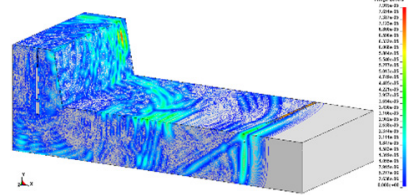

b) T2, 1/2 model

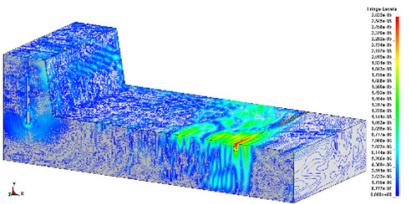

c) T3, 1/2 model

Fig. 3. The propagation of blasting seismic waves (1375 kg charge per delayed interval)

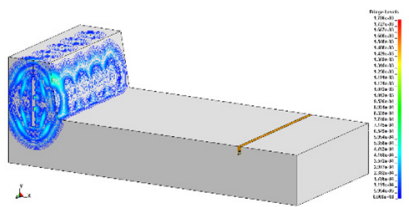

a) $\mathrm{T} 1,1 / 2$ model

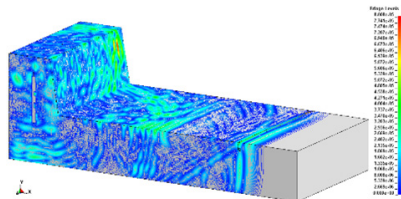

b) $\mathrm{T} 2,1 / 2$ model

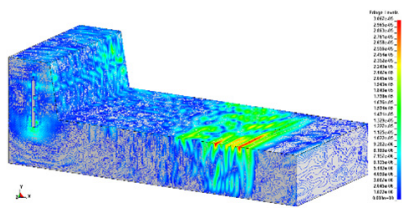

c) T3, 1/2 model

Fig. 4. The propagation of blasting seismic waves ( $2475 \mathrm{~kg}$ charge per delayed interval)

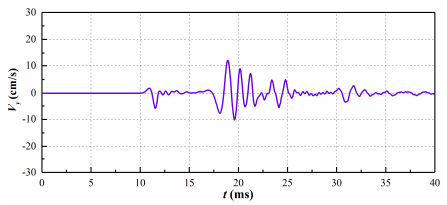

a) $275 \mathrm{~kg}$ charge

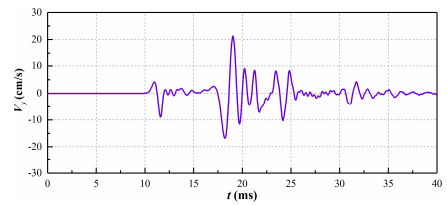

b) $1375 \mathrm{~kg}$ charge

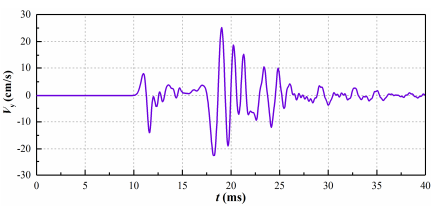

c) $2475 \mathrm{~kg}$ charge

Fig. 5. The vertical velocity history curve of the pipe under the condition of no vibration-isolating slot

The vibration peak value in each time history curve is shown in Fig. 5. The relationship between the single explosive charge and the maximum vertical vibration velocity of the pipe has been shown in Fig. 6 . The graph shows that the maximum vertical vibration speed of pipeline has a positive correlation rather than linear correlation with the amount of homogeneous explosive charge. This is in accordance with the trend reflected by the prediction formula of blasting seismic peak value which is proposed by the national standard "GB6722-2014". 


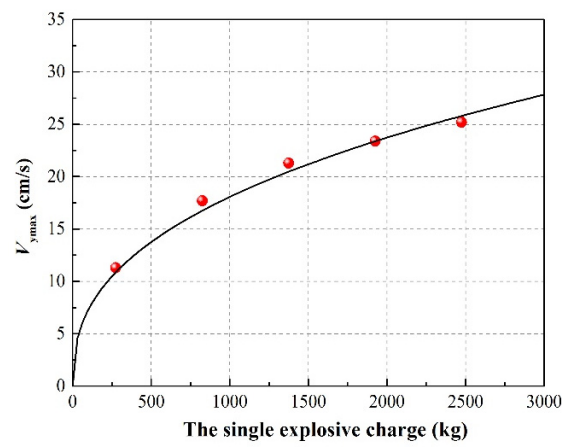

Fig. 6. The relationship between the single explosive charge and the maximum vertical vibration velocity of the pipe

In order to research the relationship between the depth of vibration-isolating slot and the vibration-isolating effect, under the condition that vibration-isolating slot is $3.0 \mathrm{~m}$ deep, the vibration velocity distribution map is shown in Fig. 7 and Fig. 8. The number of holes for a single explosive charge is only 1 hole or 9 holes which are represented here, and the other is no longer described. Taking $275 \mathrm{~kg}$ and $2475 \mathrm{~kg}$ homogeneous explosive charge respectively, the comparison of vibration waveform between the extracted pipeline with $3.0 \mathrm{~m}$ vibration-isolating slot and that without vibration-isolating slot has been shown in Fig. 9.

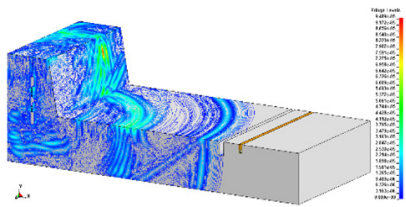

a)

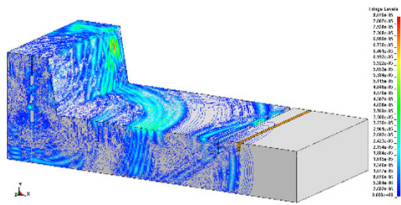

b)

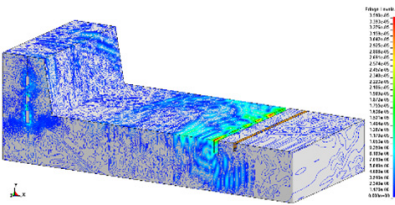

c)

Fig. 7. Isolation effect of vibration-isolating slot on blasting seismic wave propagation

(275 kg charge per delayed interval, $3.0 \mathrm{~m}$ depth)

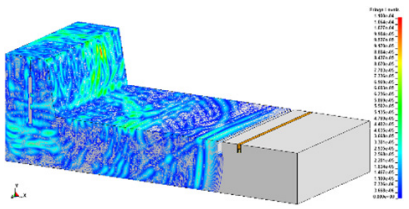

a)

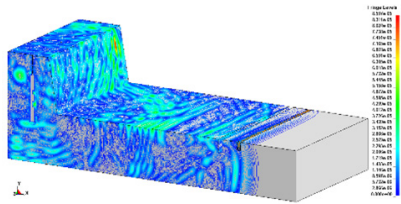

b)

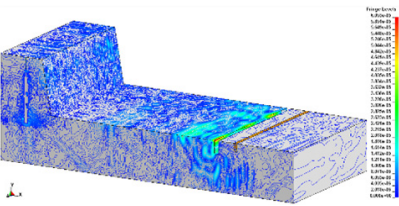

c)

Fig. 8. Isolation effect of vibration-isolating slot on blasting seismic wave propagation ( $2475 \mathrm{~kg}$ charge per delayed interval, $3.0 \mathrm{~m}$ depth)

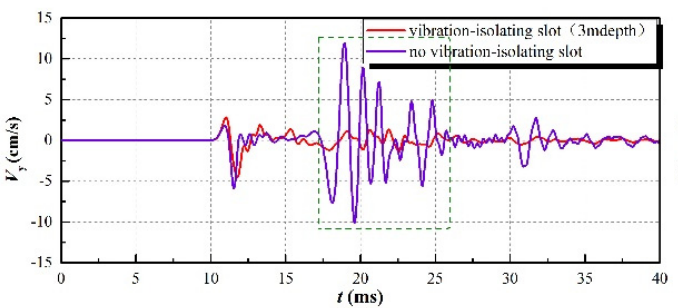

a) $275 \mathrm{~kg}$ charge per delayed interval

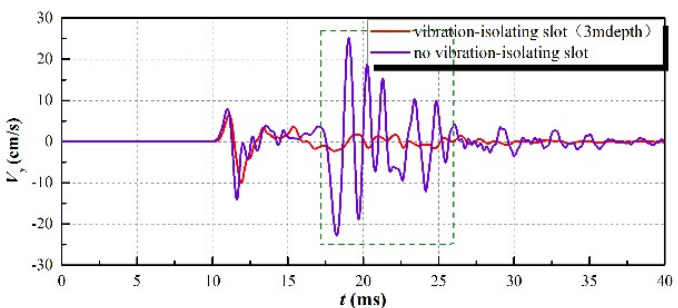

b) $2475 \mathrm{~kg}$ charge per delayed interval

Fig. 9. The clipping effect of vibration-isolating slot on the blasting seismic wave

According to the diagram, it is implied in the vertical vibration speed history curve that the vibration speed of the pipeline with vibration-isolating slot has a larger decrease than that without 
slot as a whole. In particular, the vibration curve of the dotted line in the figure shows that the vibration isolating effect of vibration-isolating slot on the shock wave is obvious. In order to analyze the influence rule of the vibration-isolating slot depth on the vibration-isolating effect, it extracts and computes the numerical results of the other slots, and calculates the vibration-isolating ratio.

Based on the above numerical results, the peak velocity $V_{y \max }$ and the vibration-isolating ratio $\lambda$ of the vertical vibration in various working conditions were discussed. The calculation results show that, whether it is $275 \mathrm{~kg}$ homogeneous explosive charge or $2475 \mathrm{~kg}$ homogeneous explosive charge, the vibration-isolating rate of the vibration-isolating slot increases along with the increase of the depth of the vibration-isolating slot, which is consistent with the research results.

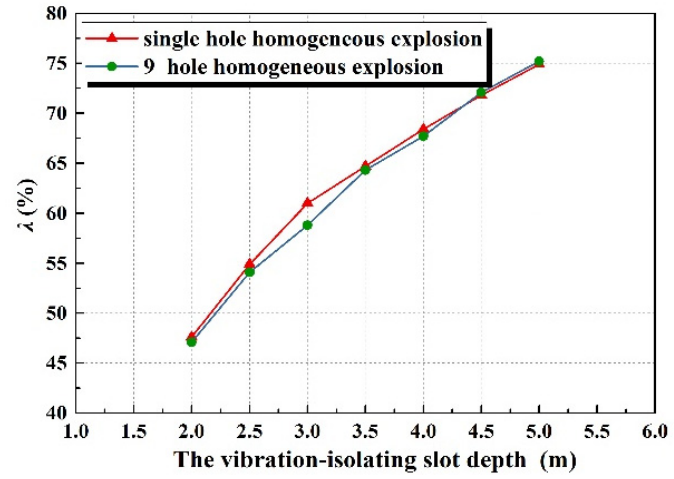

a)

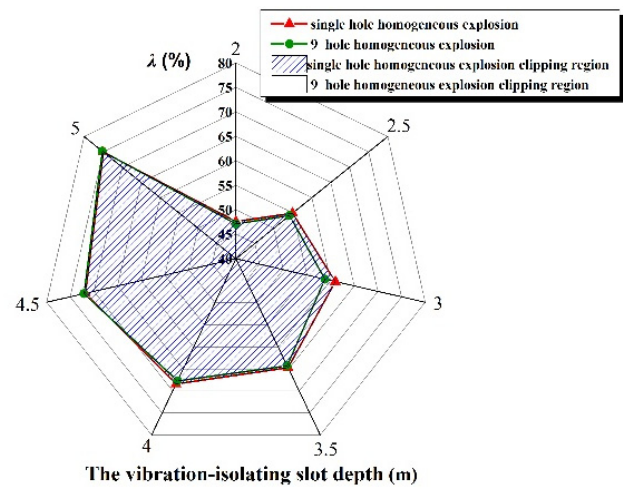

b)

Fig. 10. The vibration-isolating ratio $\lambda$ with the depth of different vibration-isolating slot

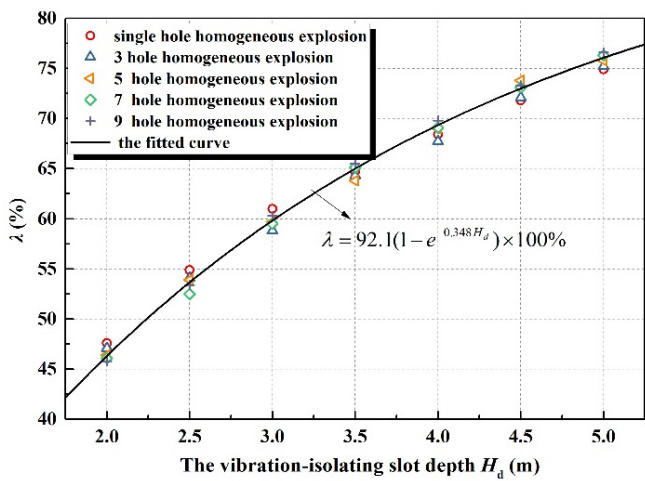

Fig. 11. The vibration-isolating ratio $\lambda$ with the depth of different vibration-isolating slot

In addition, we found a valuable rule: two kinds of homogeneous explosive charge in different working conditions are different, but the vibration-isolating ratio is very close in vibration-isolating slot with same depth $H_{d}$. The calculation data based on $275 \mathrm{~kg}$ homogeneous explosive charge ( 1 hole homogeneous explosion), $1375 \mathrm{~kg}$ homogeneous explosive charge (5 hole homogeneous explosion) and $2475 \mathrm{~kg}$ homogeneous explosive charge ( 9 hole homogeneous explosion) has been shown in Fig. 11.

It can be seen from the above diagram that the amount of homogeneous explosive charge (homogeneous detonating holes) in several working conditions is different, but the vibration-isolating ratio is quite close if vibration-isolating slot structure parameter, the depth $H_{d}$, is same, which should be the essence of physical mechanics phenomenon. Considering the practicability in engineering applications and the convenience to formulate norms for relevant departments, fit the data and the fitting formula expresses as the followed: 
$\lambda=92.1\left(1-\mathrm{e}^{-0.348 H_{d}}\right) \times 100 \%$,

where vibration-isolating ratio is $\lambda$, depth of vibration-isolating slot is $H_{d}, e$ is 2.718 .

\section{Conclusions}

1) Nonlinear dynamic finite element analysis model can well simulate dynamic response of the geotechnical field subjected to millisecond blasting. Simple and practical, the numerical method can be selected and adjusted according to different research objectives and content, which will provide technical support for the research on the influence of millisecond blasting on the vibration-isolating effect of vibration-isolating slot.

2) The vibration speed of the pipeline with vibration-isolating slot has a larger decrease than that without slot as a whole. The homogeneous explosive charge in different working conditions are different, but the vibration-isolating ratio is very close in the vibration-isolating slot with same structure parameter. The millisecond blasting is hardly affected by total explosive charge. But the blasting seismic intensity is influenced by explosive charge in each stage directly.

\section{Acknowledgements}

This research was financially supported by the National Nature Science Foundation of China, Nos. 51678567, 11102233 and 51608530.

\section{References}

[1] Wu Cong-Shi, Gao Xiao-Chu Earthquake effect of millisecond blasting in large area. Journal of Rock Mechanics and Engineering, Vol. 15, 1996, p. 529-532.

[2] Lin Da-Chao, Zhang Qi Experimental study on shallow ground vibration of double source millisecond blasting. Engineering Explosion, Vol. 6, Issue 4, 2000, p. 1-5.

[3] Anderson D. A., Winzer S. R., Ritter A. P. On computer-aided seismic analysis and discrimination. Proceedings of 3rd International Symposium, 1987, p. 247-261.

[4] Gao Wen-Xue, Jin Qian-Kun Engineering practice and numerical simulation of millisecond blasting in deep-hole grooves. Explosion and Impact, Vol. 19, Issue 2, 1999, p. 164-169.

[5] Hewetra Numerical prediction of tunnel crater blasting and bench blasting. Technology Corporation, 2012, p. 117-124.

[6] Ls-Dyna Vibration of Use Manual. Version 971/Release 4, Livermore SoftWare Technology Corporation, Vol. 37, Issue 10, 2013, p. 475-482.

[7] Li Xi-Bing, Ling Tong-Hua Response spectrum analysis of ground vibration induced by single deck and multi-deck blasting. Journal of Rock Mechanics and Engineering, Vol. 24, Issue 14, 2005, p. 2409-2414. 